\title{
Estudio comparativo en la erradicación del Helicobacter pylori: ranitidina-citrato bismuto frente omeprazol más dos antibióticos durante 7 días
}

\author{
L. BUJANDA, A. SÁNCHEZ, C. IRIONDO, A. SANTOS, A. COSME*, C. MUÑOZ \\ Servicio de Digestivo. Hospital San Eloy. Baracaldo. Vizcaya. \\ *Servicio de Digestivo. Hospital Ntra. Sra. de Aránzazu. San Sebastián
}

\begin{abstract}
RANITIDINE BISMUTH CITRATE VERSUS OMEPRAZOLE PLUS TWO ANTIBIOTICS FOR HELICOBACTER PYLORI ERADICATION
\end{abstract} DURING ONE WEEK

\section{RESUMEN}

Objetivos: Comparar la eficacia en la erradicación del Helicobacter pylori con ranitidina-citrato bismuto $(\mathrm{RB})$ frente al tratamiento clásico con omeprazol.

Material y métodos: Se estudiaron de forma prospectiva y randomizada la eficacia de erradicación en 200 pacientes consecutivos con dos pautas de tratamiento; OCA-7 (omeprazol $20 \mathrm{mg} / 12$ horas, amoxicilina $1 \mathrm{~g} / 12$ horas, claritromicina $500 \mathrm{mg} / 12$ horas durante 7 días) y RBCA-7 (RB $400 \mathrm{mg} / 12$ horas, amoxicilina $1 \mathrm{~g} / 12$ horas, claritromicina 500 $\mathrm{mg} / 12$ horas durante 7 días). La erradicación se valoró con el test del aliento al menos 4 semanas de finalizar el tratamiento.

Resultados: 112 hombres y 88 mujeres con una media de edad de 48 años (18-82 años). 100 pacientes eran randomizados a OCA-7 y 100 pacientes a RBCA-7. En total 186 pacientes completaban todo el estudio. La tasa de erradicación por intención a tratar en el grupo OCA-7 fue del $72 \%$ y del grupo RBCA-7 del 73\%. La tasa de erradicación por protocolo en el grupo OCA-7 fue del 78\% y del grupo RBCA-7 del 77\% ( $\mathrm{p}=0,3)$. Cinco pacientes del grupo OCA-7 y 9 pacientes del grupo RBCA-7 relataron efectos secundarios, 3 de estos últimos suspendieron el tratamiento por diarrea.

Conclusiones: Ranitidina citrato bismuto más dos antibióticos es tan eficaz y segura como el tratamiento clásico con omeprazol en la erradicación del Helicobacter pylori.

PALABRAS CLAVE: Ranitidina-citrato bismuto. Helicobacter pylori. Erradicación.

\section{ABSTRACT}

Aims: To compare the efficacy of ranitidine bismuth citrate $(R B)$ combined with two antibiotics during seven days in comparison with OCA-7 for treatment of Helicobacter pylori.

Methods: In a randomized clinical trial 200, consecutive patients infected by Helicobacter pylori were studied prospectively. One of two regimens was given for 7 days; OCA-7 (omeprazole $20 \mathrm{mg}$ b.i.d., amoxi cillin $1 \mathrm{gr}$ b.i.d., clarithromycin $500 \mathrm{mg}$ b.i.d.) or RBCA (RB 400 b.i.d., amoxicillin $1 \mathrm{gr}$ b.i.d., clarithromycin $500 \mathrm{mg}$ b.i.d.). Eradication was defined as a negative breath test one month after therapy.

Results: 112 male and 88 female with a mean age of 48 years (range 18-82). 100 patients were randomized to OCA-7 and 100 patients to RBCA-7. Overall 186 patients completed the treatment. Intention to-treat eradication was in the group OCA-7 of $72 \%$ and $73 \%$ in group RBCA-7. Per-protocol eradicatication was in the group OCA-7 of 78\% and 77\% in group RBCA-7 $(p=0,3)$. Five patients in the group OCA-7 and nine in the group RBCA-7, had side effects, three of these patients did not com plete therapy by diarrhea.

Conclusions: RB with two antibiotics is as effective and safe as OCA-7 for the eradication of Helicobacter pylori.

KEY WORDS: Ranitidine bismuth citrate. Helicobacter pylori. Eradica tion.

L. Bujanda, A. Sánchez, C. Iriondo, A. Santos, A. Cosme, C. Muñoz. Estudio comparativo en la erradicación del Helicobacter pylori: ranidinacitrato bismuto frente omeprazol más dos antibióticos durante 7 días. An Med Interna (Madrid) 2001; 18: $361-363$.

\section{INTRODUCCIÓN}

El número de pacientes que reciben tratamiento erradicador para el Helicobacter pylori (HP) es cada vez mayor (1). Muchas pautas han sido propuestos la mayoría de ellas recomiendan un inhibidor de la bomba de protones (IBP) asociado a dos antibióticos, generalmente en nuestro país, claritromicina y amoxicilina durante 7 días (2). Otra pauta también aceptada en nuestro país pero menos extendida es el empleo de los dos antibióticos anteriores, asociados a ranitidina citrato bis- muto (RB) (2). Con cualquiera de estas pautas se consiguen tasas de erradicación superiores al $70 \%(3,4)$. En nuestra experiencia y dentro de estudios multicéntricos internacionales hemos obtenido unas tasas de erradicación con omeprazol, claritromicina y amoxicilina durante 7 días de un $73 \%$ frente a un $85 \%$ obtenido con RB, claritromicina y amoxicilina durante 7 días (5).

Nuestro objetivo era comparar en nuestro medio la tasa de erradicación del HP con RB más dos antibióticos frente al tratamiento clásico.

Trabajo aceptado: 20 de Febrero de 2001

Correspondencia: Luis Bujanda. C/ Ocharán Mazas, 13 Q-1A. 39700 Castro Urdiales 


\section{MATERIAL Y MÉTODOS}

Se realizó un estudio prospectivo y randomizado en 200 pacientes consecutivos para valorar la tasa de erradicación con dos pautas diferentes: OCA-7 (omeprazol $20 \mathrm{mg} / 12$ horas, amoxicilina 1g/12 horas, claritromicina $500 \mathrm{mg} / 12$ horas durante 7 días) y RBCA-7 (RB $400 \mathrm{mg} / 12$ horas, amoxicilina $1 \mathrm{~g} / 12$ horas, claritromicina $500 \mathrm{mg} / 12$ horas durante 7 días). Los comprimidos eran administrados después de las comidas

En todos pacientes se confirmó la presencia de HP mediante el test de la ureasa rápida y/o examen histológico (hematoxilina eosina) de al menos dos biopsias de antro gástrico o por medio del test del aliento $\mathrm{C}_{13}$ o $\mathrm{C}_{14}$.

Los pacientes eran excluidos si presentaban estenosis pilórica, obstrucción o dificultad al vaciado gástrico, neoplasias del tracto digestivo superior, esofagitis, embarazo, lactancia, tratamiento previo erradicador, menores de 18 años, pacientes con tratamiento activo o reciente con AINEs o antibióticos, enfermedades crónicas severas (como por ejemplo, cardiopatía, insuficiencia renal o hepática), coagulopatía y alergia a algunos de los componentes estudiados. Los pacientes daban el consentimiento informado al estudio.

La efectividad del tratamiento erradicador era valorada a partir de las 4 semanas de finalizar el tratamiento con el test del aliento $\mathrm{C}_{13}$ o $\mathrm{C}_{14}$. Durante este periodo era permitido la utilización de antiácidos y anti-H2 para los síntomas. La eficacia de la erradicación era valorada por la intención a tratar (todos los pacientes independientemente de que no completasen el estudio, estos últimos eran considerados como fracaso del tratamiento) y por protocolo (excluyendo los pacientes que iniciaban el estudio pero que no lo completaban).

\section{Análisis estadístico}

La comparación entre los dos tratamientos se realizó por el test Chi-cuadrado y analisis multivariable (regresión logística múltiple). La significación estadística era considerada para una $\mathrm{P}<0,05$.

\section{RESULTADOS}

Un total de 200 pacientes participaban en el estudio (112 hombres y 88 mujeres) con una media de edad de 48 años (1882 años). 100 pacientes eran randomizados a OCA-7 y 100 pacientes a RBCA-7. Las características de los pacientes están reflejadas en la tabla I. Ocho pacientes del grupo OCA-7 y 6
TABLA I

\begin{tabular}{lcc}
\multicolumn{3}{c}{ TABLA I } \\
\multicolumn{3}{c}{ CARACTERÍSTICAS DE LOS PACIENTES } \\
\hline & OCA-7 & RBCA-7 \\
\hline Edad media (años) & 50 & 46 \\
Sexo $(\mathrm{H} / \mathrm{M})$ & $55 / 45$ & $57 / 43$ \\
Peso $(\mathrm{Kg})$ & 68 & 69 \\
Altura $(\mathrm{cm})$ & 166 & 167
\end{tabular}

pacientes del grupo RBCA-7 no volvían a la consulta tras indicar el tratamiento. En total, 186 pacientes completaban todo el estudio.

La tasa de errradicación por intención a tratar en el grupo OCA-7 fue del $72 \%$ y del grupo RBCA-7 del 73\%. La tasa de erradicación por protocolo en el grupo OCA-7 fue del 78\% y del grupo RBCA-7 del $77 \%(p=0,3)$. No hubo diferencias estadísticamente significativa en función de la indicación de la erradicación, en algunos casos, debido al escaso número de pacientes como en la dispepsia no ulcerosa (Tabla II).

Cinco pacientes del grupo OCA-7 relataron efectos secundarios, en tres pacientes gingivitis y/o mal sabor de boca, en uno astenia y en otro diarrea. Ninguno de ellos suspendió la medicación por los mismos. Del grupo RBCA-7 presentaron 9 efectos secundarios, en 7 diarrea que cedió al suspender o finalizar el tratamiento y 2 mal sabor de boca. De los 9 pacientes, 3 suspendieron el tratamiento por diarrea.

\section{DISCUSIÓN}

La RB es un compuesto con actividad frente al HP debido a la capacidad antisecretora de la ranitidina que facilita la acción de otros antibióticos ,y por otro lado, al efecto protector de la mucosa y al efecto bactericida de las sales de bismuto $(3,4)$.

En un metaanalisis realizado por Gisbert y cols. (3) observan como la erradicación con RBCA era del 76,6\% mientras que los IBP, claritromicina y amoxicilina era del 73,7\%. El riesgo relativo de la primera pauta era de 1,15 frente a 1 de IBP-CA durante 7 días. Cuando se combinaba RB con metronidazol y claritromicina por intención a tratar era de $87,2 \%$ mientras que para IBP era del $74,9 \%$ con un riesgo relativo para la primera pauta de 1,76 (3). La mejor respuesta con metronidazol se da fundamentalmente en otros países donde la tasa de resistencias al metronidazol son menores que en nuestro país (3).

Existen únicamente tres estudios publicados, no resúmenes (Tabla III) que comparan ambas pautas, se observa como

TABLA II

ERRADICACIÓN DEL HELICO BACTER PYLO RI CON OCA-7 Y RBCA-7 SEGÚN LA INDICACIÓN

\begin{tabular}{|c|c|c|c|c|c|c|}
\hline & \multicolumn{3}{|c|}{ OCA-7 } & \multicolumn{3}{|c|}{ RBCA-7 } \\
\hline Indicación & Pacientes & IT & PP & Pacientes & IT & PP \\
\hline Dispepsia tipo ulcus & $3-2 P$ & $60 \%(5)$ & $100 \%$ & $2+6-1 P$ & $67 \%(9)$ & $75 \%$ \\
\hline Gastritis & $10-31+4 P$ & $69 \%(45)$ & $76 \%$ & $11+32-1 P$ & $73 \%(44)$ & $74 \%$ \\
\hline Ulcus gástrico & $3+9$ & $75 \%(12)$ & $75 \%$ & $3+7-$ & $70 \%(10)$ & $70 \%$ \\
\hline Ulcus duodenal & $3+15-1 P$ & $79 \%(19)$ & $83 \%$ & $1+12-3 P$ & $75 \%(16)$ & $92 \%$ \\
\hline Duodenitis & $4+14-1 P$ & $74 \%(19)$ & $78 \%$ & $4+16-1 P$ & $76 \%(21)$ & $80 \%$ \\
\hline
\end{tabular}

-; test del aliento negativo +; test del aliento positivo P; pacientes perdidos o abandono del tratamiento IT; intención a tratar PP; por protocolo 


\section{TABLA III}

ESTUDIOS PREVIOS CO M PARATIVO S ENTRE OCA-7 Y RBCA-7

$\begin{array}{cccc}\text { IT - 0CA-7 } & \text { IT - RBCA-7 } & \text { PP - OCA-7 } & \text { PP - RBCA-7 } \\ \% & \% & \% & \% \\ \text { (n-pacientes) (n-pacientes) } & & \end{array}$

\begin{tabular}{lcccc}
\hline Sung y cols. & & & & \\
$1998(6)$ & $89,6 \%(50)$ & $90(50)$ & $87,5 \%$ & $94 \%$ \\
$\begin{array}{l}\text { Catalano y cols. } \\
1998(7)\end{array}$ & $75 \%(39)$ & $76 \%(42)$ & $88 \%$ & $84 \%$ \\
$\begin{array}{l}\text { Castellote y Porta } \\
2000(8)\end{array}$ & $66 \%(49)$ & $77 \%(49)$ & $73 \%$ & $85 \%$ \\
$\begin{array}{l}\text { Bujanda y cols. } \\
2001\end{array}$ & $72 \%(100)$ & $73 \%(100)$ & $78 \%$ & $77 \%$ \\
\hline
\end{tabular}

$\mathrm{IT}$; intención a tratar PP; por protocolo UD; úlcera duodenal en dos de ellos hay una mejor tasa de erradicación con RBCA7 , y en otro peor, pero sin observar en ninguno de ellos diferencias estadísticamente significativas.

Cuando comparamos ambas pautas según el tipo de indicación observamos como no existe diferencias significativas, no siendo valorable en la dispepsia debido al bajo número de pacientes ( 5 y 9 pacientes en cada grupo). Otros autores han observado una mejor tasa de erradicación de RBCA en la dispepsia no ulcerosa (9).

Los efectos secundarios eran similares con ambos regímenes, destacando la diarrea con RBCA-7 probablemente debido a la RB. En todos los pacientes se resolvió al suspender o tras finalizar el tratamiento. Estos mismos efectos secundarios han sido previamente observados en otros estu$\operatorname{dios}(3,4)$.

En resumen, podemos decir que tanto la pauta con OCA-7 como la pauta con RBCA-7 son igual de eficaces y seguras en la erradicación del HP.

\section{Bibliografía}

1. EHPSG. Current European concepts in the management of Helicobacter pylori infection. The Maastricht Consensus Report. Gut 1997; 41:8-13

2. Sainz Sametier R, Borda F, Domínguez E, Gisbert JP y Gruipo Conferencia Española de Consenso. Conferencia Española de Consenso sobre la infección por helicobacter pylori. Rev Esp Enf Digest 1999; 91:777-84.

3. Gisbert JP, Gonzalez L, Calvet X, Roque M, Gabriel R, Pajares JM. Helicobacter pyloir eradication: proton pump inhibitor vs. Ranitidine bismuth citrate plus two antibiotics for 1 week a meta-analysis of efficacy. Aliment Pharmacol Ther 2000; $14 ; 1141-50$.

4. Van Oijen AH, Verbeek AL, Jansen JB, De Boer WA. Review article: treatment of Helicobacter pylori infection with ranitidine bismuth citrate or porton pump inhibitor based triple therapies. Aliment Pharmacol Ther 2000; 14:991-9.

5. Herrerías JM, Bujanda L, Pena D, Chaves-da-Cruz A, Fueyo A, Compañy T. Efficacy and cost study in Portugal and Spain of three different 7

day eradication regimens of Helicobacter pylori. Gastroenterology 1999 ; 116:A186.

6. Sung JY, Leung WK, Ling TK, Yung MY, Chan KL, Lee YT, et al. Oneweek use of ranitidine bismuth citrate, amoxycillin and clarithromycin for the treatment of Helicobacter pylori-related duodenal ulcer. Aliment Pharmacol Ther 1998; 12:725-30.

7. Catalano F, Catnazaro R, Bentivegna C, Brogna A, Condorellli G, Cipolla R. Ranitidine bismuth citrate versus omeprazole triple therapy for the eradication of Helicobacter pylori and healing of duodenal ulcer. Aliment Pharmacol Ther 1998; 12:59-62.

8. Castellote J, Porta F. La asociación de ranitidina-citrato de bismuto con dos antibióticos en la erradicación de Helicobacter pylori. Med Clin (Barc) 2000; 115:118-9.

9. Gisbert JP, Carpio D, Marcos S, Gisbert JL, García Gravalos R, Pajares JM. One-week therapy with pantoprazole versus ranitidine bismuth citrate plus two antibiotics for Helicobacter pylori eradication. Eur J Gastroenterol Hepatol 2000; 12:489-95. 